\title{
KULEUVEN
}

\section{Conference scheduling - a personalized approach}

Vangerven B, Ficker A, Goossens D, Passchyn W, Spieksma F, Woeginger G. 


\title{
Conference Scheduling - A Personalized Approach ${ }^{\text {th }}$
}

\author{
Bart Vangerven ${ }^{\mathrm{a}, \mathrm{b}, *}$, Annette M.C. Ficker ${ }^{\mathrm{a}}$, Dries R. Goossens ${ }^{\mathrm{b}}$, Ward Passchyn ${ }^{\mathrm{d}}$, Frits C.R. Spieksma ${ }^{\mathrm{a}}$, \\ Gerhard J. Woeginger ${ }^{c}$ \\ ${ }^{a}$ Faculty of Economics and Business, KU Leuven, Naamsestraat 69, 3000 Leuven, Belgium. \\ ${ }^{b}$ Faculty of Economics and Business Administration, Ghent University, Tweekerkenstraat 2, 9000 Ghent, Belgium. \\ ${ }^{c}$ Faculty of Mathematics, Computer Science and Natural Sciences, RWTH Aachen, Ahornstr. 55, 52056 Aachen, Germany. \\ ${ }^{d}$ OM Partners, Koralenhoeve 23, 2160 Wommelgem, Belgium.
}

\begin{abstract}
Scientific conferences have become an essential part of academic research and require significant investments (e.g. time and money) from their participants. It falls upon the organizers to develop a schedule that allows the participants to attend the talks of their interest. We present a combined approach of assigning talks to rooms and time slots, grouping talks into sessions, and deciding on an optimal itinerary for each participant. Our goal is to maximize attendance, taking into account the common practice of session hopping. On a secondary level, we accommodate presenters' availabilities. We use a hierarchical optimization approach, sequentially solving integer programming models, which has been applied to construct the schedule of the MathSport (2013), MAPSP (2015) and ORBEL (2017) conferences.
\end{abstract}

Keywords: conference scheduling, computational complexity, case study, integer programming

\section{Introduction}

Conferences are an essential aspect of (academic) research, as they allow researchers to present their work and receive feedback, as well as to learn from 5 attending talks, poster sessions, or discussion panels. There is also a social aspect to conferences: they can lead to meetings and collaborations networking remains critical. However, conferences require a considerable effort in terms of time (e.g. 10 preparing talks, traveling time) and money (e.g. registration fees, traveling expenses, hotels) from their participants, and have a nonnegligible environmental impact [2]. In fact, there is some debate about the value of scientific conferences, see 15 e.g. [3]. Obtaining exact figures with respect to the amount of money involved in organizing scientific conferences seems difficult; it is written in [4] that "an estimate of more than 100.000 medical meetings per year may not be unrealistic ... the cumu-

\footnotetext{
An extended abstract corresponding to an earlier version of this paper appeared in [1].

${ }^{*}$ Corresponding author.
}

to fathom". Note that this figure applies to medical conferences alone.

Given these investments, it is the responsibility of the organizers to develop a schedule that allows 25 participants to maximally benefit from participating. Or, making this concrete, the schedule should enable participants to attend the talks of their interest. This clearly benefits speakers as well, potentially increasing both the size and the level of interest of their audience. Typically, a conference schedule groups talks into sessions (a set of talks taking place consecutively in the same room); consecutive sessions are separated by a break. Furthermore, the vast majority of conferences feature several sessions taking place at the same moment in time, i.e., sessions are scheduled in parallel. Consequently, a participant may be confronted with times where several attractive talks compete for his/her attendance (i.e., a scheduling conflict), while at other times (s)he finds nothing of interest in the schedule.

One popular approach to schedule conferences is track segmentation [5]. The organizer groups talks that cover a similar topic or method into tracks or 45 clusters, which are then assigned to a room and 
scheduled in parallel. Note that a track can consist of multiple sessions. If a participant were only interested in talks from a single track, then (s)he 100 can stay in that track's room for the duration of the conference without experiencing any scheduling conflict. However, apart from difficulties in forming meaningful clusters, track segmentation is not very effective if the participant's preferences are diverse, and not restricted to one particular topic.

${ }_{55}$ In this work, a participant is expected to provide a list of preferred talks, which he or she would like to attend. Our goal is to develop a conference schedule that maximizes the participants' satisfaction. Primarily, this means we want to avoid schedul-

60 ing conflicts, thereby maximizing total attendance. Next, as a secondary goal, we want to minimize session hopping. Indeed, confronted with multiple talks of interest scheduled in different sessions, a participant is forced to move between several ses65 sions in order to attend as many of his or her preferred talks as possible. We call this phenomenon session hopping, and its presence is a clear indication of the existence of strong preferences of participants. Session hopping can be perceived as disturb70 ing by presenters and their audiences. Moreover, the session hopper still tends to miss parts of the preferred talks, due to the time it takes to switch 120 rooms and presenters not always starting at exactly the scheduled time. Finally, motivated by practical 75 considerations, we also take presenter availabilities into account.

Our main contribution is the description of a 125 method for the planning of a (scientific) conference. Based on given preferences of the participants, our so method schedules individual talks in order to maximize total attendance; this is in contrast to many other approaches that work on the level of sessions or streams. As a secondary, original criterion, we take session hopping into account, aiming for schedules that allow participants to stay within the same room during a session. We are the first to incorporate session hopping in our scheduling approach, as 135 session hopping is either assumed to be forbidden or non-existing in the literature, as opposed to regular o participant practice. Our method has been used to schedule three scientific conferences, namely MathSport 2013, MAPSP 2015, and ORBEL2017 — we ${ }_{140}$ give a detailed account of our experience with the method.

We provide an overview of related work in Section 2. A detailed problem definition, is given in Section 3, followed by computational complexity ${ }_{145}$ results in Section 4. Next, we describe our solution method in Section 5. Finally, we present case studies on the MathSport 2013, MAPSP 2015, and ORBEL 2017 conference in Section 6. We finish with conclusions in Section 7.

\section{Literature review}

Thompson [6] discerns two approaches to conference scheduling: a presenter-based perspective (PBP) and an attender-based perspective (ABP). With a PBP, the main goal is to meet time preferences and availability restrictions of the presenters. On the other hand, from an ABP, participants' preferences are solicited, in order to maximize their satisfaction. In the rest of this section, we will first discuss contributions that focus on the $\mathrm{PBP}$, continue with papers that follow an ABP, and conclude with a few papers that solve subproblems of conference scheduling.

\subsection{Presenter-based perspective}

Potthoff and Munger [7] discuss a problem where sessions need to be assigned to time periods (rooms are ignored). The authors assume that the clustering of talks into sessions has already been done, in a way that each session belongs to a subject area. The goal is to find a schedule that spreads the sessions for each subject area among the time slots as evenly as possible, ensuring that no presenter has other duties (e.g. being discussant) in simultaneous sessions. An IP formulation is presented and applied to a problem instance extracted from a past meeting of the Public Choice Society, including 96 sessions and over 300 participants. This problem is revisited by Potthoff and Brams [8], who extend the IP formulation to take into account presenter availabilities. Furthermore, their method is applied to schedule two Public Choice Society meetings, with 76 and 45 sessions.

Edis and Sancar Edis [9] consider a very similar problem, but at the level of talks instead of sessions. Each talk has a given topic, and should be assigned to a session and a time period, such that all talks in each session have the same topic, and the occurrence of simultaneous sessions with the same topic is minimized. Furthermore, the number of talks in different sessions with same topic should be balanced, and some talks cannot be scheduled simultaneously. The authors also discuss an extended setting where presenters have preferred and 
non-preferred days. An IP formulation is presented, which is used to solve a hypothetical instance, including 170 talks on one of 10 topics, to be scheduled into sessions of at most 5 talks, over 12 time 200 periods.

Nicholls [10], like Potthoff and Munger [7], also assumes that papers have been assigned to sessions beforehand by the organizers, but includes room assignment. The problem at hand is to assign each 205 to a room and a time period, such that no presenter is scheduled at two sessions simultaneously. The goal is to maximize the number of presenter preferences (e.g. preferred day or time slot) met. Participant preferences are not elicited, but 210 be included implicitly by the program chair, for instance by allocating appropriate rooms to sessions based on expectations regarding attendance. The author presents an algorithm, which is essentially a step-wise constructive heuristic, complemented 215 165 with a set of rules to accommodate preferences and resolve conflicts. Nicholls [10] applied his method to schedule a Western Decision Sciences Institute annual conference. This conference had over 300 participants, involving over 80 sessions and span- 220

\subsection{Attender-based perspective}

An early attempt to optimize participant satisfaction is by Eglese and Rand [11], who collect a 225 list of 4 preferred sessions (and one reserve ses175 sion) from each participant. In their conference scheduling problem, sessions need to be assigned to time periods and rooms such that the sum of the weighted violations of session preferences is mini- 230 mized. Furthermore, sessions can be offered multiple times, a decision which is also part of the problem. Although the number of rooms is limited and some rooms are not equipped with the right facilities for some sessions, room capacity is assumed to 235 be always sufficient. The paper reports the schedul185 ing of the national Tear Fund conference, including 15 distinct sessions, over 4 time periods and 7 rooms. As an IP formulation for a problem of this size was deemed intractable at the time, the prob- 240 lem was solved using simulated annealing.

Sampson and Weiss [12] extend the Eglese and Rand [11] setting as they consider rooms with finite seating capacities. They present a heuristic procedure that simultaneously assigns session of- 245 ferings to time periods and rooms, and decides for dure is tested on a number of randomly generated problem instances. Sampson [5] describes how an annual meeting of the Decision Sciences Institute with 213 sessions to be scheduled over 10 time slots was handled using this method. Nearly half of the 1086 registered participants submitted ranked preferences for talks, which was used to rank the sessions. A post-conference survey revealed that about one quarter of the participants found the resulting schedule "much better" than in previous meetings. The method is also a part of a simulation to numerically address other issues that might be faced by a conference organizer. For instance, Sampson and Weiss [13] discuss tradeoffs between the length of the conference, the number of offerings per session and participant satisfaction. They also investigate how seating capacity, room availability, and the utilization of time slots impact participant satisfaction.

Gulati and Sengupta [14] enhance the problem description by Sampson and Weiss [12] by augmenting the objective function with a prediction of the popularity of a talk, based on reviewers' assessments of the submissions and linked with time slot preferences of participants (e.g. late and lastday time slots are often poorly attended). The overall goal is to maximize the total session attendance. Gulati and Sengupta [14] develop a solution method called TRACS (TRActable Conference Scheduling), which is essentially a greedy algorithm; no empirical results or computational analysis are reported.

The conference scheduling problem discussed by Thompson [6] is also similar to that of Sampson and Weiss [12]. However, in [6], meeting rooms may have different capacities, and may not always be available. He presents a method that employs a constructive heuristic followed by a simulated annealing procedure. The author performs a number of computational experiments, based on randomly generated data as well as data from a real, yet unspecified, conference. The latter includes 47 distinct sessions (some of which were to be offered 2 or 3 times), 8 time slots, and 8 rooms with different capacities. Presenters present in 1 to 5 sessions and each of the 175 participants have provided between 0 and 8 preferred sessions (neither ranked nor weighted). The author finds that his heuristic outperforms randomly as well as manually generated schedules.

Le Page [15] assumes that each participant provides a list with a given number of sessions he or 
she wishes to attend. This allows to create a consents the number of participants that wish to attend both sessions $i$ and $j$. The problem is to assign the sessions to time slots and rooms (with differ- 305 ent capacities), such that the sum of conflicts beween simultaneous sessions is minimized. Furthermore, sessions with the same topic must be assigned to the same room, and some sessions need to be planned consecutively on the same day. The author 310 develops a semi-automated heuristic in four steps, which is used to schedule a meeting of the American Crystallographic Association. This meeting includes 35 sessions, to be assigned to 5 rooms and 7 time periods. Months before the conference, preferences were solicited from the 1100 participants; about $10 \%$ of them provided a list of 7 preferred 315 sessions. Most popularity predictions based on this input turned out to be accurate during the actual conference.

Ibrahim et al. [16] focus on a conference scheduling problem where talks need to be assigned to time 320 slots (spread over a number of days) in 3 parallel tracks. Each talk belongs to a field, and the schedule should be such that talks of the same field do not occur simultaneously. Furthermore, it should be avoided to schedule talks belonging to the same ${ }^{325}$ pair of fields in parallel more than once on the same day. The authors discuss construction methods, based on results from combinatorial design theory, for 3 cases. One case is based on data from the National Conference in Decision Science and includes 330 73 sessions, belonging to 8 fields, to be scheduled over 26 time slots and 2 days. Note that this setting does not involve grouping talks into sessions. Moreover, the sequence of the talks within a track one day is of no importance, and all talks from 335 the same field can be swapped without changing the solution quality.

In the so-called preference conference optimization problem (PCOP) as defined by Quesnelle and

290 Steffy [17], talks need to be assigned to a time slot 340 and a room, such that scheduling conflicts are minimized. Furthermore, room and presenter availabilities need to be taken into account, including the fact that some presenters are involved in more than one talk and must be able to attend each one of 34 them. Some talks are required to be offered multiple times. Quesnelle and Steffy [17] show that PCOP is NP-hard and discuss an IP formulation, together with a number of performance considera300 tions such as symmetry reduction. They apply their 350 method on a problem instance, based on a PenguiCon conference with 253 talks. As no individual participant preferences were available, the authors have randomly generated this data from historical attendance data, for various choices of the standard deviation of the number of preferred talks per participant. Notice that the issue of grouping talks into sessions is not included in this problem, in fact, as in Ibrahim et al. [16], each talk could be seen as a session.

\subsection{Related problems}

The problem of grouping talks into coherent sessions, given one or more keywords for each talk, is discussed by Tanaka et al. [18] and Tanaka and Mori [19]. The objective function is a non-linear utility function of common keywords, with the underlying idea that papers in the same session have as many common keywords as possible, provided that the number of talks is balanced over the sessions. This problem is tackled using Kohonen's self-organizing maps [18] and a hybrid grouping genetic algorithm [19]. Both methods are tested on data from a conference of the Institute of Systems, Control and Information Engineers in Japan with 313 papers and 86 keywords.

Zulkipli et al. [20] ignore session coherence as they attempt to group talks into equally popular sessions. The underlying idea is that in a setting with rooms of similar size and assuming that session hopping is forbidden, this will maximize participants' satisfaction in terms of seating capacity. Given a weight for each talk, based on preferences from the participants, the goal is to assign talks to sessions, such that the sum of the talk weights is balanced over the sessions. The authors present a goal programming method, which is applied to one case, involving 60 talks to be grouped into 15 sessions.

Martin [21] elaborates on the sessions selection problem for the participant, given the conference schedule. He develops a decision support system for participants to determine their itinerary. Using a web-based approach, keyword preferences are elicited and matched with keywords supplied by talks, in order to produce an aggregate rating for each talk. This approach, which does not involve an optimization algorithm, has been used for a conference of the UK Academy of Information Systems. About one third of the 118 participants made use of the decision support system, however, the author 
was not able to predict session attendance based on the keyword ratings.

\section{Problem Description}

There are a number of crucial ingredients in our

problem. First, there is a set of talks that needs to be scheduled; the set of talks is denoted by $X$. ${ }^{405}$ Second there is a set of timeslots, denoted by $T$; a timeslot refers to a period in time during which a number of talks are held in parallel — we assume that the number of talks that are held in parallel (i.e. the number of parallel sessions) is given and we denote that number by $n$. Further, we assume without loss of generality that the number of talks $|X|$ is a multiple of $n$ (this can be achieved by adding my talks). A final ingredient of our problem are the participants, denoted by the set $P$, and their ${ }^{415}$ profiles.

Definition 1. A profile of a participant $p \in P$ is represented by a binary vector $q(p)$ where $q(p)_{i}$ equals 1 if and only if participant $p$ wishes to attend ${ }_{42}$ talk $i \in X$. A profile consisting of only 0 entries is called a trivial profile.

In other words, a profile represents the preferences of a participant. All these ingredients allow us to formally state our problem. We assume that talks that are held in parallel cannot both be attended by the same participant, and vice versa, i.e., talks that are assigned to distinct timeslots can be attended by the same participant. The attendance of a talk denotes the number of participants attending a talk, and total attendance refers to the summed attendances over the talks. We assume that a participant, when a preferred talk is scheduled, will attend this talk, and in case multiple preferred talks presented at the same time, (s)he will arbitrarily choose one of these talks to attend. Finally, we assume all participants attend the entirety of the conference.

Definition 2. Given the participants' profiles $390 q(p)$, and given the number of parallel sessions $n$, the Conference Scheduling Problem with $n$ parallel sessions (CSP- $n$ ) seeks to assign every talk to a timeslot such that each timeslot receives exactly $n{ }^{440}$ talks, while maximizing total attendance.

The profiles allow us to compute the parameter $v_{i}:=\sum_{p \in P} q(p)_{i}$ : the number of participants who wish to attend talk $i$. Notice that this number can ${ }_{445}$ be realized in case there are no parallel sessions, i.e. when $n=1$

From a computational complexity standpoint, CSP- $n$ is an NP-hard optimization problem, already in case of three parallel sessions. We address this issue in more detail in Section 4.

It is clear that there will be several optimal solutions to CSP- $n$, as the grouping of the parallel talks into sessions and their order within a session do not impact attendance. Hence, as a secondary goal, we aim to minimize the total number of session hops. This will settle the composition of the sessions, taking into account the talks that are to be scheduled in parallel in order to maximize attendance. However, the resulting schedule still leaves room to decide during which timeslots these sessions are scheduled. This gives freedom to accommodate potential restrictions on the availabilities of speakers. Thus, in a final phase, we assign the (parallel) sessions to timeslots, minimizing the number of violated presenter availabilities. Concluding, the resulting conference scheduling problem has three objectives: maximizing attendance (5.1), minimizing session hopping (5.2), and satisfying presenter availabilities (5.3), which are considered hierarchically in this order. Notice that we have not taken room capacities into account; in Section 6 we describe how, if necessary, this issue can still be dealt with.

\section{Computational complexity of CSP-n}

In the following two theorems, we respectively show that the CSP-2 is polynomially solvable, and that the CSP- $n$ is NP-hard for $n \geq 3$.

Theorem 1. CSP-2 is solvable in polynomial time.

Proof. We reduce CSP-2 to a minimum weight perfect matching problem, which is polynomially solvable [22]. Given a graph $G=(V, E)$, a matching $G$ is a set of pairwise non-adjacent edges. A perfect matching is a matching which matches all nodes of $G$, i.e., every node is incident to exactly one edge of the matching.

The reduction goes as follows. Given an instance of CSP-2, we construct a complete, edgeweighted, graph $G=(V, E)$ such that each talk in CSP-2 corresponds to exactly one node in $G$; thus $V:=X$. For every distinct pair of talks $i$ and $j \in X$, we calculate a coefficient $c_{i, j}$ capturing how much attendance is missed if both talks 
$i$ and $j$ are planned simultaneously, i.e., we set $c_{i, j}:=\left|\left\{p \in P: q(p)_{i}=q(p)_{j}=1\right\}\right|$. Since (i) any solution to CSP-2 can be regarded as $\frac{|X|}{2}$ pairs of talks, and hence is a perfect matching, and (ii) the ${ }_{490}$ coefficient $c_{i, j}$ equals the missed attendance when talks $i$ and $i$ are planned simultaneously, the result follows.

Theorem 2. CSP-n is NP-hard for each fixed $n \geq{ }_{495}$ 3 .

Proof. We will prove that the decision variant of CSP-3, which asks the question "Given a number of talks and a set of participants with corresponding profiles, does a schedule consisting of 3 parallel ses- 500 sions exist such that no attendance is missed?", is NP-complete. To prove this, we will use the Triangle Partition Problem (TPP), which is known to be NP-complete even for graphs with a maximal degree of at most four [23]. An instance of the TPP is a graph $G=(V, E)$ with $|V|=3 \ell$, where $\ell \in \mathbb{N}_{0}^{+}$. A triangle is a collection of three nodes in $G$ such that each pair is connected by an edge. The question that TPP asks is then: "Can the nodes of $G$ be partitioned into $\ell$ disjoint sets $V_{1}, V_{2}, \ldots, V_{\ell}$ each containing exactly 3 nodes, such that each of these $V_{i}$ is the node set of a triangle in $G$ ?".

We transform an arbitrary instance of TPP into ${ }_{510}$ an instance of the CSP-3. Each node in $G$ will correspond to a talk in CSP-3, i.e. $X:=V$. We define $P:=\{(i, j): i, j \in V, i \neq j,(i, j) \notin E\}$. Next, for all $p \in P$, say $p=(i, j)$, we have

$$
q(p)_{x}= \begin{cases}1 & \text { if } x=i \text { or } x=j \\ 0 & \text { otherwise }\end{cases}
$$

This completely specifies an instance of CSP-3.

Suppose we have a yes-instance of TPP, then $\ell$ disjoint sets exist each containing exactly 3 vertices. These vertices correspond to three parallel talks as follows: the talks corresponding to the nodes in the ${ }^{52}$ triangle are scheduled simultaneously; parallel talks are assigned to timeslots in any order. Note that no participant misses a talk, because of the connected triangles; edges correspond to talks that can be scheduled together without missing any attendance. ${ }^{525}$ Thus, a yes-instance of CSP-3 is obtained. Suppose we have a yes-instance of the decision variant of CSP-3, then we know which talks are in parallel. From this, we can find a partition into triangles; simply select the nodes corresponding to the paral- 530 lel talks as the nodes of a triangle. These nodes correspond to triangles, because otherwise there would have been missed attendance. From this it follows that CSP- $n$ is NP-hard for $n \geq 3$.

Note that this complexity result is tight, in the sense that CSP- $n$ is NP-hard even if each participant has only 2 preferred talks in his/her profile (and the problem becomes trivial if each participant has at most one preferred talk). Furthermore, our result strengthens the result that the preference conference optimization problem (PCOP) is NP-hard by Quesnelle and Steffy [17]. Indeed, their result is based on the presence of room and presenter availabilities, while in CSP- $n$ every talk can be allocated to any timeslot.

\section{Method}

In this section we will explain a hierarchical three-phased approach to scheduling conferences. In the first phase (see Section 5.1), we maximize total attendance, based on the participants' profiles, i.e., we solve CSP- $n$. In the second phase, we seek to minimize the number of session hops, given that total attendance is maximal (see Section 5.2). Finally, in a third phase, we take into account presenter availabilities. We do this by minimizing the number of violated availability constraints, while fixing the total attendance and number of session hops at the levels obtained in the previous two phases (see Section 5.3).

\subsection{Phase 1: maximizing total attendance}

It should be obvious that maximizing total attendance is equivalent to minimizing total missed attendance. Informally, it is best to avoid scheduling talks that are on a same profile in parallel. Let $H$ denote the set of all $n$-tuples consisting of distinct talks, $H \subseteq X^{n}$. For each $e \in H$, we set $c_{e}:=\sum_{p \in P} \max \left\{0, \sum_{i \in e} q(p)_{i}-1\right\}$. In words: the coefficient $c_{e}$ denotes the total missed attendance if the talks in the $n$-tuple $e$ are scheduled in parallel. Notice that the coefficients $c_{e}$ are in fact a generalization of the conflict matrix used in [15]. Indeed, the conflict matrix indicates the missed attendance at the level of a session if two talks are scheduled in parallel sessions, while our coefficient does the same for any $n$ parallel talks.

Next, we set up an integer programming model using the binary variable $x_{e}$ which is 1 if and only 
if all talks in $n$-tuple $e$ are planned in parallel.

$$
\begin{aligned}
& \operatorname{Min} \sum_{e \in H} c_{e} x_{e} \\
& \text { s.t. } \sum_{e \in H: i \in e} x_{e}=1 \quad \forall i \in X \\
& x_{e} \in\{0,1\} \\
& \forall e \in H
\end{aligned}
$$

Clearly, the objective function, Equation 1, minimizes missed attendance. The first set of constraints, Equation 2, ensures that every talk is included in exactly one $n$-tuple. Finally, Equation 3 the variables $x_{e}$ are binary. Note that he number of variables in this formulation amounts to $\left(\begin{array}{c}|X| \\ n\end{array}\right)$, which is a potential bottleneck.

\subsection{Phase 2: minimizing session hopping}

Recall that an $n$-tuple refers to $n$ talks that will

ake place in parallel. Phase 1 gives us $\frac{|X|}{n}$ such $n$-tuples. Here, in phase 2, our goal is to assemble the $n$-tuples into so-called $k$-blocks.

Definition 3. A $k$-block consists of a ordered set of $k$ ordered $n$-tuples, yielding $n$ parallel sessions, ${ }^{595}$ each session consisting of $k$ consecutive talks.

Consider a set of $k n$-tuples found in phase 1 , say $e_{1}, e_{2}, \ldots, e_{k}$. Clearly, there are different ways to organize a set of $k n$-tuples into a $k$-block: one can permute the sequence of $n$-tuples $e_{1}, e_{2}, \ldots$, $e_{k}$, and one can permute the $n$ talks within each $n$-tuple $e_{i}$. In total this gives $k !(n !)^{k}$ possibilities, i.e., given $k n$-tuples there are $k !(n !)^{k}$ distinct $k$ blocks corresponding to it. We now describe how we select a $k$-block for a given set of $k n$-tuples. For times a participant with a particular profile needs to switch between different sessions in order to attend the maximum number of talks in this $k$-block he/she is interested in. More precise, we define the

560 hopping number of a participant in a $k$-block as the minimum number of session hops needed by that participant to attend the maximum number of preferred talks in that $k$-block. Given a profile, and a $k$-block, we can compute this profile's hopping numnumber of profiles as examples. The top left example shows that the participant is interested in the first and last talk in session 1. The hopping number for this profile will therefore be 0 , as indicated

1 and not miss any of his/her preferred talks. The top right example shows the profile of a participant interested in the first talk in session 2 and the last talk in session 1. In order to attend both talks, this participant will have to switch rooms exactly once, leading to a hopping number of 1 . There are two alternative ways this participant can switch, one is indicated using the full line, the other using the dashed line. Similarly, in the bottom left example, a participant with this profile will have to switch exactly twice to attend all talks of his or her interest, as indicated by the full line. The final example, on the bottom right, shows a profile and a $k$-block where at a particular moment in time, more than 5 one talk of interest is planned. In that case, we assume that the participant chooses talks such that he/she can attend the maximum number of talks of his/her interest, while minimizing the number of required session switches. In the bottom right example this means that the participant will choose to stay in session 1 for the second talk, as indicated by the full line, instead of switching to session 3 and then back to session 1. Thus, given a particular profile and a $k$-block we can compute its hopping number, and next, we find the hop coefficient of a $k$-block by summing the corresponding hopping numbers over all participants.

For each set of $k n$-tuples, we compute a $k$-block $b$ that minimizes the hop coefficient. The resulting value is denoted by $w_{b}$ and represents the total number of session switches that will result from having this block $b$ as part of the conference schedule. We define $B(k)$ as the set of $k$-blocks. Conferences often use multiple values for $k$, meaning that a conference features sessions with different numbers of consecutive talks in a session; typically the value of $k \in\{2,3,4\}$.

We now build an integer programming model to minimize the number of session hops (given that we have found the $n$-tuples maximizing attendance). Let $\mathcal{B}=\cup_{k} B(k)$, further, we write $e \in b$ to denote that block $b$ contains $e$ as an $n$-tuple. The parameter $r_{k}$ corresponds to the number of $k$-blocks required in the schedule. The binary variable $y_{b}$ equals 1 if and only block $b$ is included in the sched- 

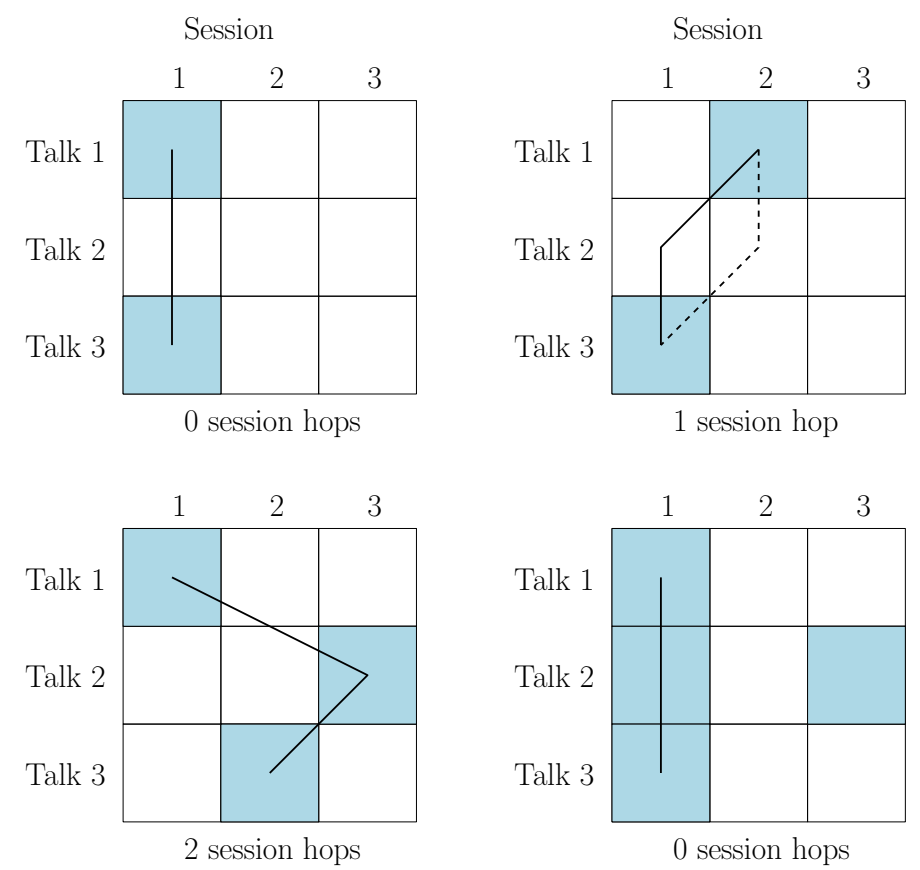

Figure 1: Session hopping examples: 3-blocks with profiles of a single participant.

ule.

$$
\begin{array}{lr}
\operatorname{Min} \sum_{b \in \mathcal{B}} w_{b} y_{b} & \\
\text { s.t. } & \sum_{b \in B(k)} y_{b}=r_{k} \\
\sum_{b \in \mathcal{B}: e \in b} y_{b}=1 & \forall e \in H \\
y_{b} \in\{0,1\} & \forall b \in \mathcal{B}
\end{array}
$$

The objective function, Equation 4, minimizes the number of hops over all possible $k$-blocks. The first constraints, Equation 5, ensure that we select the proper number of each $k$-block. Equation 6 makes certain that every $n$-tuple (input from phase 1 ) is used exactly once. Finally, Equation 7 enforces that our decision variables $y_{b}$ are binary.

After phase 2 we have composed the $k$-blocks that, given $n$-tuples that maximize attendance, minimize session hopping. Once we have the $k$ blocks, it is easy to see how a personalized optimal itinerary can be constructed for every participant. By constructing the $k$-blocks for the second phase, we already know the maximum number of preferred talks each participant can attend in every $k$-block, as well as how many session hops are required in order to actually attain that attendance. As a result, ticipants and present each participant an individual itinerary.

\subsection{Phase 3: presenter availabilities.}

In this phase, we assign the blocks found in Phase 2 to timeslots while minimizing the number of violated speaker availabilities. As the order of the talks within a block has been settled, this phase will assign each selected $k$-block, and hence each talk, to a timeslot. We define $T_{S} \subseteq T$ as the set of timeslots that correspond to session starting times. The number of violated availabilities if block $b$ is assigned to timeslot $t \in T_{S}$ is denoted by $u_{b, t}$, and can easily be computed from known presenters availabilities. We use an assignment based integer programming formulation where $z_{b, t}=1$ if block $b$ is scheduled to start in timeslot $t \in T_{S}$, and 0 otherwise.

$$
\begin{array}{lr}
\operatorname{Min} \sum_{b, t} u_{b, t} z_{b, t} & \\
\text { s.t. } \sum_{b} z_{b, t}=1 & \forall t \in T_{S} \\
\sum_{t} z_{b, t}=1 & \forall b \in B \\
z_{b, t} \in\{0,1\} & \forall b \in B, t \in T_{S}
\end{array}
$$

The objective function, Equation 8, minimizes the total number of violated availabilities. The first 
set of constraints, Equation 9, ensures that every timeslot at which sessions start gets assigned one block. The second set of constraints, Equation 10, ensures that every block is assigned exactly once. 675 Finally, Equation 11 enforces our variables $z_{b, t}$ to be binary.

\section{Practical applications}

In this section we will apply our three-phased conference scheduling approach to three practical cases: MathSport International (2013), MAPSP (2015), and ORBEL (2017). These are mediumsized conferences, where the number of parallel sessions equalled 2, 3, and 4 respectively. For each of these conferences, we sent an e-mail to all registered participants enquiring each participant for his/her profile (anonymously, when preferred). These profiles are available at the following URL: http://feb.kuleuven.be/public/NDBAE03/ csp_instances.zip. The schedule obtained by applying our method was adopted by the conference organizers in each case. Furthermore, based on the profiles, we were able to select suitable session chairs for each session. Indeed, for each session we selected chairs among the participants that had expressed an interest for a maximal number of talks in that session.

\subsection{MathSport 2013}

MathSport International is a biennial conference dedicated to all topics where mathematics and sport meet. The 4th edition was organized in Leu- ${ }^{705}$ ven (Belgium) on June 5-7, 2013 and attracted 76 talks (apart from 3 keynote talks) and 97 participants. The conference featured two parallel sessions, and consisted of five 4-blocks and six 3blocks.

Our preference elicitation resulted in 68 nontrivial profiles (a response rate of $70 \%$ ), amounting for 1279 indicated preferences in total. Some other interesting statistics can be found in Table 1 .

\begin{tabular}{cccc}
\multicolumn{3}{c}{ Table 1: MathSport profile statistics } \\
& Min & Avg & Max \\
Preferences/participant & 3 & 18.8 & 41 \\
Preferences/talk & 2 & 16.18 & 37
\end{tabular}

The first phase of scheduling MathSport, maxi- 720 mizing attendance, is an instance of CSP-2, which can be solved as a minimum weight perfect matching problem (see Theorem 1). For each pair of talks, the weight of an edge boils down to the number of participants that want to attend both talks. However, we had 4 speakers presenting two talks. These pairs of talks could obviously not be part of the matching, which we enforced by giving them a very high weight. We tackled this phase using a ${ }_{680}$ straightforward IP formulation, which was solved in a negligible computation time using Cplex 12.3. The optimal matching involved 42 scheduling conflicts, allowing the participants to attend $96.7 \%$ of the talks in their profile on average.

The MathSport 2013 conference was characterized by several presenter unavailabilities, such that 10 talks could not be scheduled on given days, and for 2 other talks only 1 particular timeslot was acceptable. Hence, we gave priority to phase 3 over phase 2, meaning that we first grouped the pairs of talks into parallel sessions, for which the starting times were already set (using a slightly modified version of formulation (8)-(11)). Again, it took Cplex 12.3 barely any computation time to optimize this assignment; the result was a timetable that did not violate any presenter unavailability.

Finally, we needed to decide for each group of paired talks to which session - the one in room A or the one in room B - the talks should be assigned, and in what order. As the capacity of both rooms was identical, minimizing session hopping was the only concern. Since there are only 8 (4) ways to organize a group of 4(3) pairs of talks into sessions, and 24 (6) different orders of these pairs within a session of $4(3)$ talks, this step could trivially be handled using complete enumeration.

\section{2. $M A P S P 2015$}

The workshop on Models and Algorithms for Planning and Scheduling Problems (MAPSP) is a biennial conference dedicated to scheduling, planning, and timetabling. The 12 th edition of MAPSP was held on June 8-12 2015 in La Roche-en-Ardenne (Belgium) and featured three parallel series of sessions, spread over five days.

Specifically for MAPSP, there were eight 3-blocks and three 2-blocks available for scheduling talks, leading to a total capacity for talks of 90 . The MAPSP program committee accepted 88 talks, to which we added 2 dummy talks (corresponding to empty spaces in the conference schedule) in order to match the capacity. 
We collected 78 nontrivial profiles from the 120 participants; the total number of indicated preferences was 1576. Some other interesting statistics can be found in Table 2 .

\begin{tabular}{cccc}
\multicolumn{4}{c}{ Table 2: MAPSP profile statistics } \\
& Min & Avg & Max \\
Preferences/participant & 1 & 20.20 & 43 \\
Preferences/talk & 2 & 17.91 & 38
\end{tabular}

The first phase of scheduling MAPSP, maximizing attendance, is an instance of CSP-3. Remembering the coefficient $c_{e}$, as defined in Section 5.1, we have for each triple (3-tuple) of distinct talks $i, j, k \in X$ the number of missed attendance if talks $i, j$ and $k$ are scheduled in parallel: $c_{i, j, k}$. Note that this is easily computed using the profiles, as indicated in Section 5.1.

We used formulation (1)-(3), which for this problem instance amounts to $\left(\begin{array}{c}90 \\ 3\end{array}\right)=117480$ variables and 90 constraints. It turned out that, using Cplex 12.5.1 (on a laptop with an Intel Core i7-4800 MP CPU @ 2.70Ghz processor and 8 GB RAM), we ${ }_{790}$ could solve this instance in less than 8 seconds; a very reasonable computation time. We obtained an (optimal) objective value of 155 . Equivalently, the obtained triples allowed the participants to attend 1421 of the 1576 preferred talks according to the profiles.

In the second phase of scheduling MAPSP, our goal is to assemble the triples into eight 3-blocks, and three 2-blocks such that session hopping is minimized. Recall that a 3-block, as well as a 2-block, consists of three parallel sessions, each taking place 800 in a different room.

Observe that the model as presented in Section 5.2, i.e. formuation formulation (4)-(7), is directly applicable for generating both 2-blocks and 3 -blocks. We could solve our second phase of the 80 755 MAPSP 2015 instance, using Cplex 12.5.1, in less than 0.5 seconds (again on a laptop with an Intel Core i7-4800 MP CPU @ 2.70Ghz processor and 8 GB RAM). The optimal objective value of the second phase is 120 , which is the total number of hops 810 for all participants.

Note that in order to arrive at a schedule, there is still freedom in the allocation of sessions to rooms. Indeed, the allocation of sessions to rooms does not influence the attendance or the number of session 815 that the rooms have infinite capacity. The available rooms at the MAPSP conference each had a different (and finite) capacity: these capacities equalled 770170,100 and 40 seats. So, with the three sessions of each 3- and 2-block known, we used the following strategy to allocate sessions to rooms. First, find in each session the talk with the largest number of votes. The session for which this number is minimal 775 goes to the smallest capacitated room. Next, for the two remaining sessions, we sum the votes, and put the session with the largest sum in the largest room. This system of allocation offers no hard guarantee that the room capacities are respected. However, it turned out that, using the system described above, room capacity was not an issue.

Finally, in the third phase of scheduling MAPSP, we need to identify a talk with a speaker, and take into account the various availabilities of speakers, in order to assign 2-blocks and 3-blocks to timeslots. In total, 13 speakers had availability restrictions (i.e. not being available on certain days). There were 5 presenters who were unavailable to present on Monday, there was 1 presenter unavailable to present on Tuesday, and there were 9 presenters unavailable on Friday. This phase was handled by solving formulation (8)-(11), which features $11 \times 11$ variables (since the total number of 2-blocks and 3blocks equals 11). The solution resulted in a schedule respecting all speaker availabilities, which was then implemented for MAPSP 2015.

\subsection{ORBEL 2017}

ORBEL is the annual conference of the Belgian Operational Research Society, and serves as a meeting place for researchers working in Operational Research, Statistics, Computer Science and related fields. ORBEL 2017 took place February 2-3 2017 in Brussels (Belgium), and was the 31st edition of ORBEL. ORBEL featured four parallel series of sessions, spread over two days. Specifically, a total of one 2-block, two 3-blocks, and three 4-blocks were available to schedule talks, leading to a total capacity of 80 talks, which exactly corresponds to the number of accepted talks.

We collected 101 non-trivial profiles from 140 participants, leading to a total of 1200 indicated preferences. Some statistics can be found in Table 3 .

The first phase of scheduling ORBEL, which maximizes attendance, corresponds to an instance of CSP-4. This leads to a total of $\left(\begin{array}{c}80 \\ 4\end{array}\right)=1581580$ quadruples (4-tuples). However, not all these 
Table 3: ORBEL profile statistics

$\begin{array}{cccc} & \text { Min } & \text { Avg } & \text { Max } \\ \text { Preferences/participant } & 1 & 11.54 & 29 \\ \text { Preferences/talk } & 2 & 15 & 48\end{array}$

quadruples were feasible and hence needed to be filtered out. 30 out of 80 talks were classified as being 'COMEX talks'. The organizing committee requested that the schedule ensures sessions consisting of only COMEX talks, such that the number of parallel COMEX sessions is minimal. Hence, we kept quadruples that had exactly 1 or 2 COMEX

825 talks in parallel. In addition, 4 particular talks were to be grouped in an 'ORBEL Award' session. As they could not be scheduled in parallel, we filtered out all quadruples containing 2 or more ORBEL Award talks. One of the jury members of the RBEL Award also gave a talk at ORBEL, and hence could not be scheduled in parallel with ORBEL Award talks. Finally, two presenters each had two talks, which could consequently not be in the same quadruple. Finally, 9 participants could not 835 be present on Friday, which was the day the ORBEL Award took place. In other words: the talks of these participants were not scheduled in parallel to the ORBEL Award talks. In the end, we ended up with 889573 feasible quadruples.

We used formulation (1)-(3), and in less than 2 minutes Cplex 12.6.3 (on a laptop with an Intel Core i7-4800 MP CPU @ 2.70Ghz processor and 8 GB RAM) found an optimal solution with objective value 100 . In other words: participants could see 1100 of their 1200 preferred talks.

In the second phase of scheduling ORBEL, we assembled the 20 quadruples resulting from phase 1 into one 2-block, two 3-blocks and three 4-blocks in a way that minimizes session hopping, that ensures COMEX talks are together in 1 or 2 parallel sessions, and that groups the ORBEL Award talks in a single session. In a few seconds, this resulted in an optimal solution of 281, the total number of hops for all participants, such that they can at-

855 tend the maximum number of preferred talks. The most challenging aspect of the second phase was undoubtedly to determine a $k$-block with minimal session hopping for each set of $k$ quadruples (with $k \in\{2,3,4\})$; this took around 7 hours.

Finally, in the third phase, we needed to take 910 into account availabilities constraints in order to assign the various 2-, 3-, and 4-blocks to specific

time-slots. Using an assignment-based formulation, similar to the one used for MAPSP, we could find

\section{Conclusions}

In this paper, we argue that conference scheduling is an important and relevant problem. Indeed, conferences require significant investments (time, 70 money) from participants, which strongly motivates a good schedule. We identify the Conference Scheduling Problem, where the goal is to maximize total attendance based on given preferences of the speakers. This problem is shown to be easy in case 875 of two parallel sessions, and becomes NP-hard for three or more parallel sessions. The main motivation for this research however, comes from a pragmatic origin: scheduling actual conferences. We describe how we applied our three phase scheduling method to three different conferences, MathSport 2013, MAPSP 2015 and ORBEL 2017, and discuss these cases extensively.

A possible consequence of our method could be that the resulting sessions are incoherent, since their composition is based solely on participant preferences, and not on the topic of the talk. However, as the profiles of the participants tended to contain talks on similar topics, the resulting sessions were still relatively coherent.

Another concern is that the current approach does not treat all participants equally. By preferring many talks, a participant may have a larger impact on the conference schedule than a participant with a small number of preferences. This can be remedied by giving an appropriate weight to the preferences of each participant.

A final issue is the scalability of our approach. Although our method has been developed for medium-size conferences, the question arises to what extent it scales to much larger conferences. This is relevant both when eliciting participants' preferences, as well as when solving huge integer programming models. In case preference elicitation is unpractical due to the high amount of talks, our approach could be applied on the level of streams or tracks. Indeed, talks in large conferences are often from the beginning (i.e., when submitting an abstract) assigned to streams, and the conference schedule is typically based on track segmentation. As not all streams cover the full length of the conference, there would be possibilities to minimize overlap between pairs of streams that many participants 
would like to attend. Furthermore, if overlap is unavoidable, the streams could be allocated to rooms which are close to each other, facilitating session hopping. In fact, eliciting stream preferences would provide a good idea of the required room capacities for each stream.

\section{References}

[1] B. Vangerven, A. Ficker, D. Goossens, W. Passchyn, F. Spieksma, G. Woeginger, Conference Scheduling A Personalized Approach, in: Proceedings of the 11th International Conference on Practice and Theory of Automated Timetabling (PATAT-2016), 371 - 383, 2016.

[2] D. Gremillet, Paradox of flying to meetings to protect 990 the environment, Nature 455 (7217) (2008) 1175-1175.

[3] P. Grant, The dilemma of attending (or not) scientific conferences, Canadian Journal of Physiology and Pharmacology 92 (1) (2014) v-v.

[4] J. P. Ioannidis, Are medical conferences useful? And for whom?, Journal of the American Medical Association 307 (12) (2012) 1257-1258.

[5] S. Sampson, Practical Implications of Preference-Based Conference Scheduling, Production and Operations Management 13 (3) (2004) 205-215.

[6] G. Thompson, Improving Conferences through Session Scheduling, Cornell Hotel and Restaurant Administration Quarterly 43 (3) (2002) 71-76.

[7] R. Potthoff, M. Munger, Use of integer programming to optimize the scheduling of panels at annual meetings of the Public Choice Society, Public Choice 117 (1) (2003) $163-175$.

[8] R. Potthoff, S. Brams, Scheduling of panels by integer programming: Results for the 2005 and 2006 New Orleans meetings, Public Choice 131 (2) (2007) 465-468.

[9] E. Edis, R. Sancar Edis, An integer programming model for the conference timetabling problem, C.B.U. Journal of Science 9 (2) (2013) 55-62.

[10] M. Nicholls, A small-to-medium-sized conference scheduling heuristic incorporating presenter and limited attendee preferences, Journal of the Operational Research Society 58 (3) (2007) 301-308.

[11] R. Eglese, G. Rand, Conference seminar timetabling, Journal of the Operational Research Society 38 (7) (1987) 591-598.

[12] S. Sampson, E. Weiss, Increasing service levels in conference and educational scheduling: a heuristic approach, Management Science 41 (11) (1995) 1816-1825.

[13] S. Sampson, E. Weiss, Designing Conferences to Improve Resource Utilization and Participant Satisfaction, Journal of the Operational Research Society 47 (2) (1996) 297-314.

[14] M. Gulati, A. Sengupta, TRACS: Tractable Conference Scheduling, in: Proceedings of the Decision Sciences Institute Annual Meeting (DSI 2004), 3161-3166, 2004.

[15] Y. Le Page, Optimized schedule for large crystallography meetings, Journal of Applied Crystallography 29 (3) (1996) 291-295.

[16] H. Ibrahim, R. Ramli, M. Hassan, Combinatorial design for a conference: constructing a balanced three-parallel session schedule, Journal of discrete mathematical sciences \& cryptography 11 (3) (2008) 305-317.
[17] J. Quesnelle, D. Steffy, Scheduling a conference to minimize attendee preference conflicts, in: Z. Hanzálek, G. Kendall, B. McCollum, P. Šucha (Eds.), Proceedings of the 7th Multidisciplinary International Conference on Scheduling: Theory and Applications (MISTA), 379-392, 2015.

[18] M. Tanaka, Y. Mori, A. Bargiela, Granulation of Keywords into Sessions for Timetabling Conferences, in: Proceedings of Soft Computing and Intelligent Systems (SCIS 2002), 1-5, 2002.

[19] M. Tanaka, Y. Mori, A Hybrid Grouping Genetic Algorithm for Timetabling of Conference Programs, in: P. De Causmaecker, E. Burke (Eds.), Proceedings of the 4th International Conference on the Practice and Theory of Automated Timetabling (PATAT 2002), 421-440, 2002.

[20] F. Zulkipli, H. Ibrahim, A. Benjamin, Optimization Capacity Planning Problem on Conference Scheduling, in: Proceedings of the Business Engineering and Industrial Applications Colloquium (BEIAC 2013), 911-915, 2013.

[21] A. Martin, A personalised conference programme advisor, OR Insight 18 (2) (2005) 22-30.

[22] L. Lovász, M. D. Plummer, Matching Theory, vol. 29 of Annals of Discrete Mathematics, AMS Chelsea Publishing, 1986.

[23] J. M. M. van Rooij, M. E. van Kooten Niekerk, H. L. Bodlaender, Partition Into Triangles on Bounded Degree Graphs, Theory of Computing Systems 52 (4) (2012) 687-718. 
FACULTY OF ECONOMICS AND BUSINESS

Naamsestraat 69 bus 3500

3000 LEUVEN, BELGIË

tel. + 3216326612

$f a x+3216326791$

info@econ.kuleuven.be

www.econ.kuleuven.be 